\title{
An efficient quantum photonic phase shift in a low Q-factor regime
}

\author{
P. Androvitsaneas,$^{\dagger}$ A. B. Young, ${ }^{\dagger}$ J.M. Lennon, ${ }^{\dagger}$ C. Schneider,${ }^{\ddagger}$ S. Maier,${ }^{\ddagger}$ \\ J.J. Hinchliff, ${ }^{\dagger}$ G.S. Atkinson,$^{\dagger}$ E. Harbord ${ }^{\dagger}$ M. Kamp,${ }^{\ddagger}$ S. Höfling, ${ }^{\ddagger}$ J. G. \\ Rarity, ${ }^{\dagger}$ and R. Oulton ${ }^{\dagger}$
}

$\nmid$ †uantum Engineering Technology Labs, H. H. Wills Physics Laboratory and Department of Electrical \& Electronic Engineering, University of Bristol, BS8 1FD, UK

$\ddagger$ Technische Physik, Physikalisches Institut and Wilhelm Conrad Röntgen-Center for Complex Material Systems, Universität Würzburg, Am Hubland, 97474 Würzburg, Germany

\Quantum Engineering Centre for Doctoral Training, H. H. Wills Physics Laboratory and Department of Electrical and Electronic Engineering, University of Bristol, Tyndall Avenue, BS8 $1 F D$, United Kingdom

$\S S U P A$, School of Physics and Astronomy, University of St Andrews, St Andrews, KY16 9SS, United Kingdom

E-mail:

\begin{abstract}
Solid-state quantum emitters have long been recognised as the ideal platform to realize integrated quantum photonic technologies. We demonstrate that a self-assembled negatively charged quantum dot (QD) in a low Q-factor photonic micropillar is a suitable design for deterministic polarisation switching and spin-photon entanglement. We show this by measuring a shift in phase of an input single photon of at least $2 \pi / 3$. As we explain in the text, this is strong experimental proof
\end{abstract}


that input photons can interact with the emitter deterministically. A deterministic photon-emitter interaction is a viable and scalable means to achieve several vital functionalities such as single photon switches and entanglement gates. Our experimentally determined value is limited by mode mismatch between the input laser and the cavity, QD spectral fluctuations and spin relaxation. When on-resonance we estimate that up to $\sim 80 \%$ of the collected photons couple into the cavity mode and have interacted with the QD and undergone a phase shift of $\pi$.

The dramatic progress in research into quantum dots (QDs) has led to single photon sources with record efficiency and indistinguishability. ${ }^{1-4}$ However, a full quantum network also requires the means to receive photons, as input to a node, where one would either manipulate or store the information. By maximising the interaction of the QD with light, one may use the QD transition to manipulate a photonic qubit. One way to do this is to change the photon phase between 0 and $\pi$ (i.e. two orthogonal states), dependent on the state of the QD transition. By implementing this in a near perfect single sided cavity one can map this phase shift onto the linear polarisation rotation of a photon. ${ }^{5}$ Incorporating a memory into the emitter, such as the electron spin, also allows spin-photon entanglement and subsequently the generation of photonic cluster states encoded in polarization. ${ }^{5-12}$ In order for the entanglement to be generated in a way that is scalable, the QD needs to couple exclusively to a single optical mode, this is known as the "one-dimensional (1D) atom" regime. $6,13,14$

In the perfect $1 \mathrm{D}$ regime, the emitter is only capable of interacting with a single, well-defined, optical mode. What this means is that a photonic environment needs to be engineered such that the vast majority of the emitted photons couple into the photonic mode in question, for example this could be an optical cavity or waveguide. Further the light that couples from the emitter to the photonic mode then needs to be very efficiently extracted into external optics or further integrated photonic components. This whole structure is known as a 1D atom. This creates the following two overarching requirements for designing a practical device: firstly, the passive photonic structure (i.e. without the quantum emitter present) must direct the light in such a way that efficient optical coupling of the cavity to the input/output mode is facilitated. Secondly, in the active structure the quantum emitter must couple 
predominately to the well-defined photonic mode in question (e.g. cavity mode). It is only when both of these conditions are fulfilled, that the 1D system becomes a scalable quantum information processing device. In this manuscript, we present a novel approach to realise structures that operate close to the one-dimensional atom regime, ${ }^{13}$ via the use of a low Q-factor single-sided micropillar cavity.

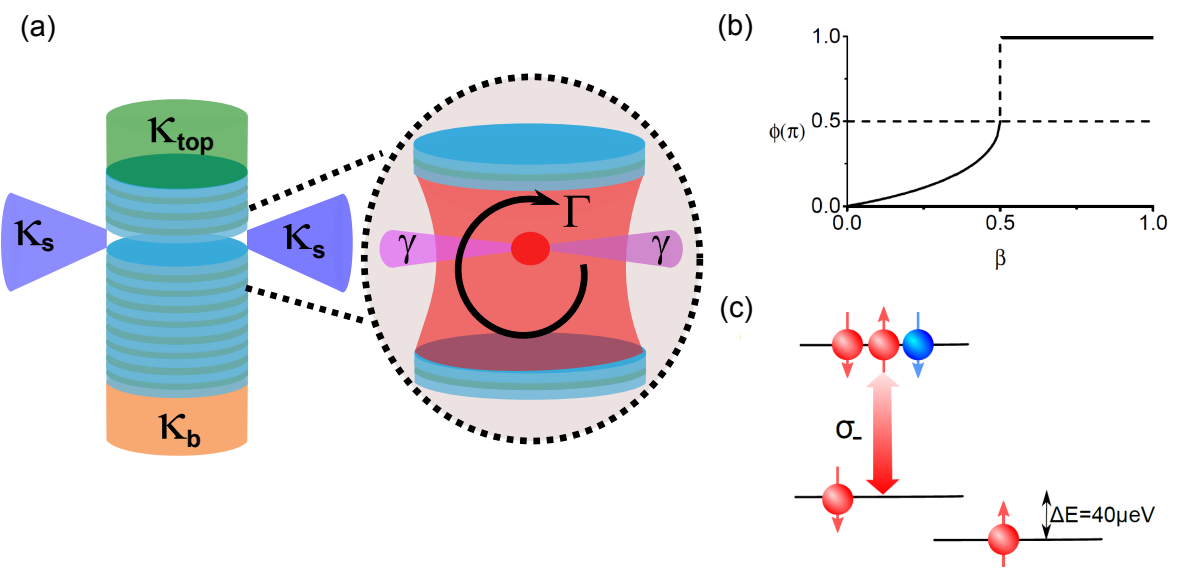

Figure 1: (a) Schematic of a QD-micropillar cavity system illustrating the decay channels for the passive device and the zoomed in region showing the relevant decay channels that define the $\beta$-factor. The green $\kappa_{t o p}$ denotes the decay channel of interest for the cavity mode in a predominantly single-sided cavity, the purple $\kappa_{s}$ denotes the decay channel for the cavity mode because of parasitic losses from the cavity mode and the brown $\kappa_{b}$ denotes the decay channel for the cavity mode through the second side of micropillar, through the substrate leakage in our case. In the zoomed in region $\Gamma$ denotes the decay rate of the QD through the cavity mode and $\gamma$ denotes the decay rate via non-cavity (leaky) modes. (b) Plot of the maximum phase shift imparted on a reflected narrow linewidth $(\ll \Gamma)$ single photon, as a function of the $\beta$-factor for $\eta_{t o p}>0.5$, according to Ref. ${ }^{14}$ The horizontal dashed line shows the $\phi=\pi / 2$ point and the vertical dashed line points to the discontinuity point occuring at $\beta=0.5$. (c) Energy level diagram of a negatively charged QD in a Faraday magnetic field. Measurements are performed on light that is resonantly scattered from the spin down transition, as shown by the transition with an arrow.

Fig. 1.(a) illustrates the parameters to be optimised in micropillar design. The passive efficiency of the photonic structure is usually parametrised as $\eta=\frac{\kappa}{\kappa+\kappa_{s}}$, where $\kappa$ is the main decay rate of the cavity mode that links the device to the external optical/photonic components. Parasitic losses that are not collected from the cavity mode are parameterised by $\kappa_{s}$. These can arise as a result of sidewall roughness, usually an issue at higher Q-factor structures ${ }^{15}$ or through lateral ridges see Ref. ${ }^{16}$ In an ideal photonic cavity $\kappa$ will dominate over $\kappa_{s}$, i.e. $\eta \sim 1$. Furthermore, in the case of a single 
sided cavity there are even more stringent requirements as now the light has to escape through only one of the two ends of the device $\left(\kappa_{t o p}\right)$. This creates a new parameter that has to be also taken into account, $\eta_{t o p}=\frac{\kappa_{t o p}}{\kappa_{t o p}+\kappa_{b}+\kappa_{s}}$, where $\kappa_{b}$ is the residual leakage of the cavity mode through the base of the micropillar (see Fig. 1(a)). For example in the case of a single sided micropillar cavity the top end is of interest and residual leakage may still exist through the bottom mirror into the substrate. ${ }^{16}$

In the active structure, i.e. when a quantum emitter is placed in the cavity mode, the device efficiency is typically parametrised by $\beta=\frac{\Gamma}{\Gamma+\gamma}$, here $\Gamma$ represents the coupling rate into the cavity mode, $\gamma$ the coupling rate into non-cavity (leaky modes). Ideally one would like to operate in the high $\beta$-factor regime where $\Gamma$ dominates over $\gamma$ (see zoomed region in Fig. 1(a)). In our case the contribution of pure dephasing (e.g. phonon scattering) is minimal, as has been shown previously. ${ }^{17}$

In order to operate close to the 1D regime then one needs maximise both $\eta$ and $\beta$. This has been shown using atom-cavity systems. ${ }^{18-21}$ High $\beta$-factor structures or even strongly coupled systems incorporating QDs are now fairly routine. , $9,16,22-32$ However, it can be challenging to realise at the same time a high $\eta$, for example having to integrate fully sources and couplers ${ }^{29}$ or place a tappered fibre on a photonic crystal waveguide to achieve this. ${ }^{28}$ In strongly coupled QD systems, typically where the Q-factor is also high, $\kappa_{s}$ becomes comparable if not larger in magnitude to $\kappa,{ }^{15,24,33,34}$ severely reducing $\eta$. As a result, more recent work has focused on the intermediate Q-factor regime (few 1000), ${ }^{22,35}$ nevertheless $\eta$ is still limited by $\kappa_{s}$. There are certain protocols ${ }^{5,36}$ that are robust to this at the expense of a reduced efficiency. In general though, low $\eta_{t o p}$ and $\beta$ factors act to not only reduce the efficiency of the device, but at the same time may introduce undesired ellipticity of the photon polarisation and subsequent loss of fidelity in most polarisation mapping or switching protocols. ${ }^{9-11}$

Another consideration in micropillar design in particular is the polarisation property of the cavity mode. The micropillar will have some small physical lateral anisotropy induced during fabrication that results in the otherwise degenerate cavity mode splitting into two orthogonally linearly polarized modes, split in energy by $\Delta$. This is typically of the order of a few $10 \mathrm{~s} \mu \mathrm{eV} .{ }^{16,35}$ Whether this has a significant effect on the polarization properties depends on the Q-factor of the cavity: if the cavity 
linewidth $\left(\kappa+\kappa_{s}\right)$ becomes comparable to $\Delta$ (i.e. if the split modes are even slightly distinguishable), the polarization of light input into the cavity mode will become distorted. For Q-factors of $\sim$ few 1000 this effect becomes significant. If one wishes to encode the photonic qubit in polarization, one requires $1 / \Gamma \gg \Delta$.

In this work we use a low Q-factor $(Q \sim 290)$ single-sided micropillar cavity. The escape rate through the mirrors is high enough that one can easily ensure that $\eta \sim 1$. At the same time a reasonably high $\eta_{t o p}(>0.9)$ is guaranteed with the highly asymmetric design of the structure (5 top mirror pairs and 18.5 bottom mirror pairs, see supporting information for sample information ${ }^{37,38}$ ). Surprisingly this can be achieved without significant impact on the $\beta$-factor. ${ }^{17}$ We achieve $\eta_{\text {top }}>0.9$ and $\beta>$ 0.5 simultaneously, which is presently only attainable in semiconductor systems by the use of such low Q-factor structure. Further to this since the spectral bandwidth of the cavity is broad (several $\mathrm{meV}$ ), any mode splitting as a result of ellipticity in the etched micropillar becomes negligible (see photoluminescence measurements in supporting information). This is crucial if one wishes to couple charged QDs to the cavity which have circularly polarised optical transitions (see Fig.1.(c)). ${ }^{16}$

The experiment we perform is to couple a narrow bandwidth laser of weak intensity efficiently into the QD micropillar device, collecting all photons scattered back from the device and measuring the phase shift induced on them in the absence of any polarisation filtering. Due to the very low intensity (weak excitation limit), single photons are scattered by the QD. Upon reflection, a narrow bandwidth $(\ll \Gamma)$ single photon will become phase shifted. The phase shift one should obtain when the photons are spectrally resonant with the QD transition is shown in Fig. 1(b), and is a rather complex function of the $\beta$-factor. For a low effciency system $(\beta<0.5)$ the maximum phase shift for such a device increases slowly with $\beta$, but never reaches more than $\pi / 2 .{ }^{35}$ However, as soon as QD coupling to the desired mode $(\Gamma)$ dominates over leaky modes $(\gamma)$, a discontinuity occurs in the plot, and at $\beta>0.5$ all photons emerging from the system have a $\pi$ phase shift (see Fig. 1), as in Ref. ${ }^{14}$ At this important transition in $\beta$-factor, the quasi-1D regime is reached. What this means is that whilst there are still losses which affect the efficiency of the device, one can deterministically (every photon will undergo 
the same phase shift) switch the photon state between two orthogonal states (phases of 0 and $\pi$ ). If the phase shift were less than $\pi$, the photon would only probabilistically end up in the $\pi$ phase shifted state, and thus filtering would be needed to post-select on those events. ${ }^{25}$ Note that in a post-selective regime one cannot use the full polarization degree of freedom to encode a qubit.

A high phase shift is not the only important parameter, for most protocols, one also requires low polarisation ellipticity of the emergent photon. The ellipticity is a rather complex function of both $\eta_{t o p}$ and $\beta$, which strongly affects the fidelity of any polarisation rotation. There are ranges for $\eta, \beta<1$, where a high fidelity polarisation rotation is possible at the expense of overall device efficiency (see supporting information, Fig. S6-S7). Practically, however, we strive to work in the limit of $\eta_{t o p} \sim 1$ and $\beta \sim 1$, where one will achieve a full rotation between two orthogonal polarisation states, with zero losses. So far direct evidence of this has not been demonstrated, due to low values for $\eta$ or $\eta_{t o p}$, which leads to ellipticity in the reflected light, limiting the phase shift to the order of $\sim \pi / 10 .{ }^{15,24}$ Recent demonstrations have exploited a low photon phase shift $(<\pi / 2)$ to probabilistically herald a $\pi$ phase on a QD spin, upon the successful detection of a rotated photon. ${ }^{25}$ Importantly, in that case one needed to post-select on rotated photons, which occurs with a low probability. We now demonstrate the next step, showing instead a $>\pi / 2$-phase switch imparted by the QD onto the photons in a deterministic interaction. In such a regime, one may generate long streams (several 10s) of photons with high probability, enough to have useful functionality, for example cluster state generation for quantum simulation circuits. ${ }^{12,39}$

\section{Results}

The experimental setup is shown in Fig. 2, similar to that in Ref. ${ }^{17}$ The sample is placed in a $0.5 T$ magnetic field along the optical axis (Faraday geometry) introduced by a permanent ring magnet, which creates a homogeneous magnetic field perpendicular to the plane of the QD. This induces an energy

splitting of $\sim 40 \mu \mathrm{eV}(\sim 26 \mathrm{pm}, 9.8 \mathrm{GHz})$ between the spin up $(|\uparrow\rangle)$ and $\operatorname{down}(|\downarrow\rangle)$ ground state 


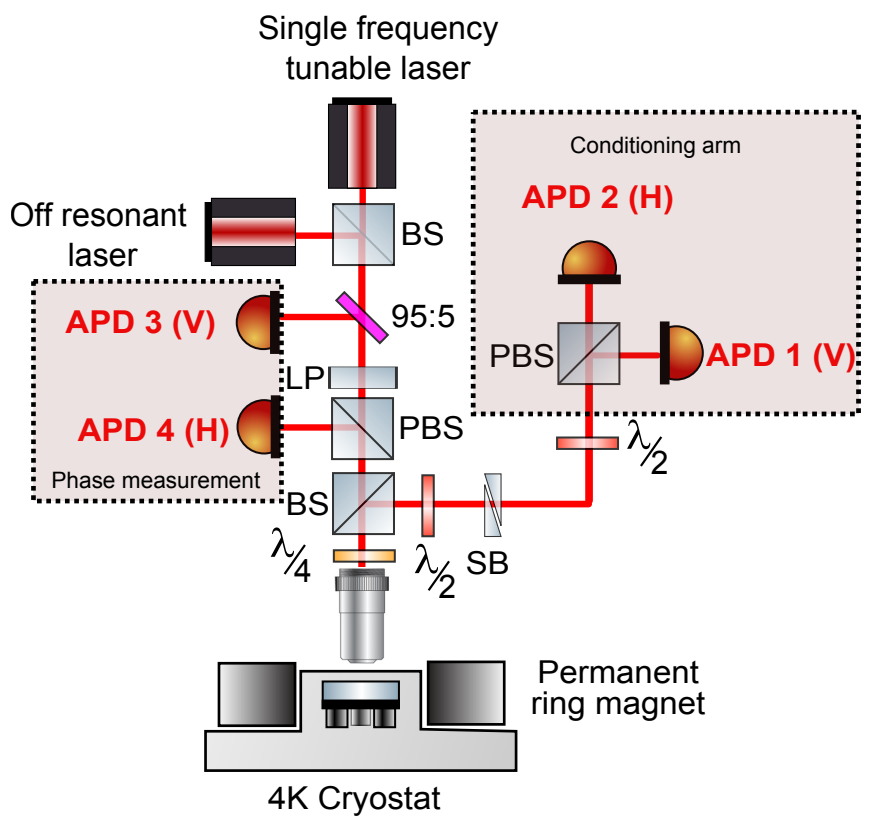

Figure 2: Experimental setup used to time resolve the QD induced phase shift. A 50 : 50 beamsplitter (BS) splits the reflected signal into two channels. One sends photons to APD-1 and APD-2 that are used as conditioning/heralding detectors, and the independently correlated phase shift is measured via APD-3 and APD-4.

(Fig.1(c)). The QD spin is not intitialised, but rather stays in a thermal state with equal probability of being spin up or spin down. The energy separation provides sufficient spin lifetime for this experiment (see supporting information). Upon measurement we project into one of the two spin states, where the system remains until it randomly flips to the opposite state (via returning into a thermal state, interaction with the nuclear spin bath or phonon scattering). Vertically linearly polarized coherent light $\left(|V\rangle=\frac{1}{\sqrt{2}}\left(\left|\sigma^{-}\right\rangle-\left|\sigma^{+}\right\rangle\right)\right)$from a single frequency laser of linewidth $<10 \mathrm{MHz}$ is input via an objective with $N A=0.7$ to the micropillar. We operate with low input power $(<0.1 \mathrm{nW}$ measured before the objective), which taken together with the narrow bandwidth ensures that we remain in the weak excitation limit, where the QD optical transitions respond linearly to the input field. The laser is spectrally tuned to be on resonance with the inhomogeneously broadened $|\downarrow\rangle \rightarrow X^{-}$transition (see Fig.1(c)), which then selectively scatters only $\left|\sigma^{-}\right\rangle$polarised light. This imposes a phase shift on only 
one circular component $\left(\left|\sigma_{-}\right\rangle\right)$of the linearly polarised photon $(|V\rangle)$, implementing the transform:

$$
|V\rangle \rightarrow|\psi\rangle=\frac{\left|r_{L}(\omega)\right| e^{i \phi(\omega)}\left|\sigma^{-}\right\rangle-\left|r_{R}(\omega)\right|\left|\sigma^{+}\right\rangle}{\sqrt{2}}
$$

where when on resonance the reflection coefficients are $\left|r_{L}\right|=|2 \eta(\beta-1)+1|$ and $\left|r_{R}\right|=|2 \eta-1|$. For the case when $\beta>0.5$, the phase shift $\phi=\pi$, if the inequality $|\eta(\beta-2)+1| / \eta \beta<0.1$ this then leads to a "giant Faraday rotation" $\left(\phi_{F R} \sim \phi / 2=\pi / 2\right)$, switching the polarisation state from $|V\rangle$ to $|H\rangle^{5}$ with fidelities in excess of 0.99 (see supporting information for more detailed discussion). One may see that this is the basis for spin-photon entanglement, which naturally ensues if one can prepare the spin into an equal superposition state: $|\psi\rangle=\frac{1}{\sqrt{2}}(|\uparrow\rangle+|\downarrow\rangle)$.

Previous measurements of this micropillar have inferred that this specific charged QD-micropillar system has a $\beta$-factor of $\sim 0.65(\Gamma \sim 0.52 \mu \mathrm{eV}(\sim 126 \mathrm{MHz}), g \sim 23.1 \mu \mathrm{eV}(\sim 56 \mathrm{MHz})$ and $\gamma \sim 0.28 \mu \mathrm{eV}(\sim 68 \mathrm{MHz}))$ with up to $\beta>0.9$ possible. ${ }^{17}$ A more detailed characterisation and study of the structure may be found in Ref. ${ }^{17}$ This device should give a $\pi$ phase shift for resonantly reflected photons according to Ref. ${ }^{14}$ (see Fig. 1(b)). However, previously we measured phase shifts of just $\sim 6^{o} .{ }^{17}$ The reduction in the measured phase shift is attributed to spectral "jitter" which leads to a shift $(\delta \omega)$ in the spectral position of the QD over a timescale shorter ${ }^{40}$ than the data acquisition time (1 sec). ${ }^{17}$ This is the source of the observed inhomogeneous broadening of the optical QD transitions. In Ref. ${ }^{17}$ a range of QD laser detunings $(\delta \omega)$ is probed during the 1s integration time, which gives rise to a much lower average photon phase shift.

Designing of novel photonic structures with electrical contacts embedded have demonstrated spectral diffusion may be overcome (not the case here). ${ }^{41,42}$ In this experiment we overcome spectral jitter in a different way. While we do not eliminate spectral jitter, we overcome it to reveal the underlying deterministic interactions by measuring the photon phase shift in $100 \mu s$ intervals (faster than the spectral jitter time, see supporting information Fig. S10). This technique allows us to demonstrate that a large phase shift is possible in these micropillars. Using a two-channel conditioning technique, 
we detect those time-windows when the QD remains close to resonance with the laser and statistics are accumulated to measure the phase shift of light reflected from the QD-micropillar system. This is only possible due to the high brightness of the system (large $\beta$-factor, and high $\eta$ ), where we count on average $1 \mathrm{MHz}$ rates on the detector at saturation.

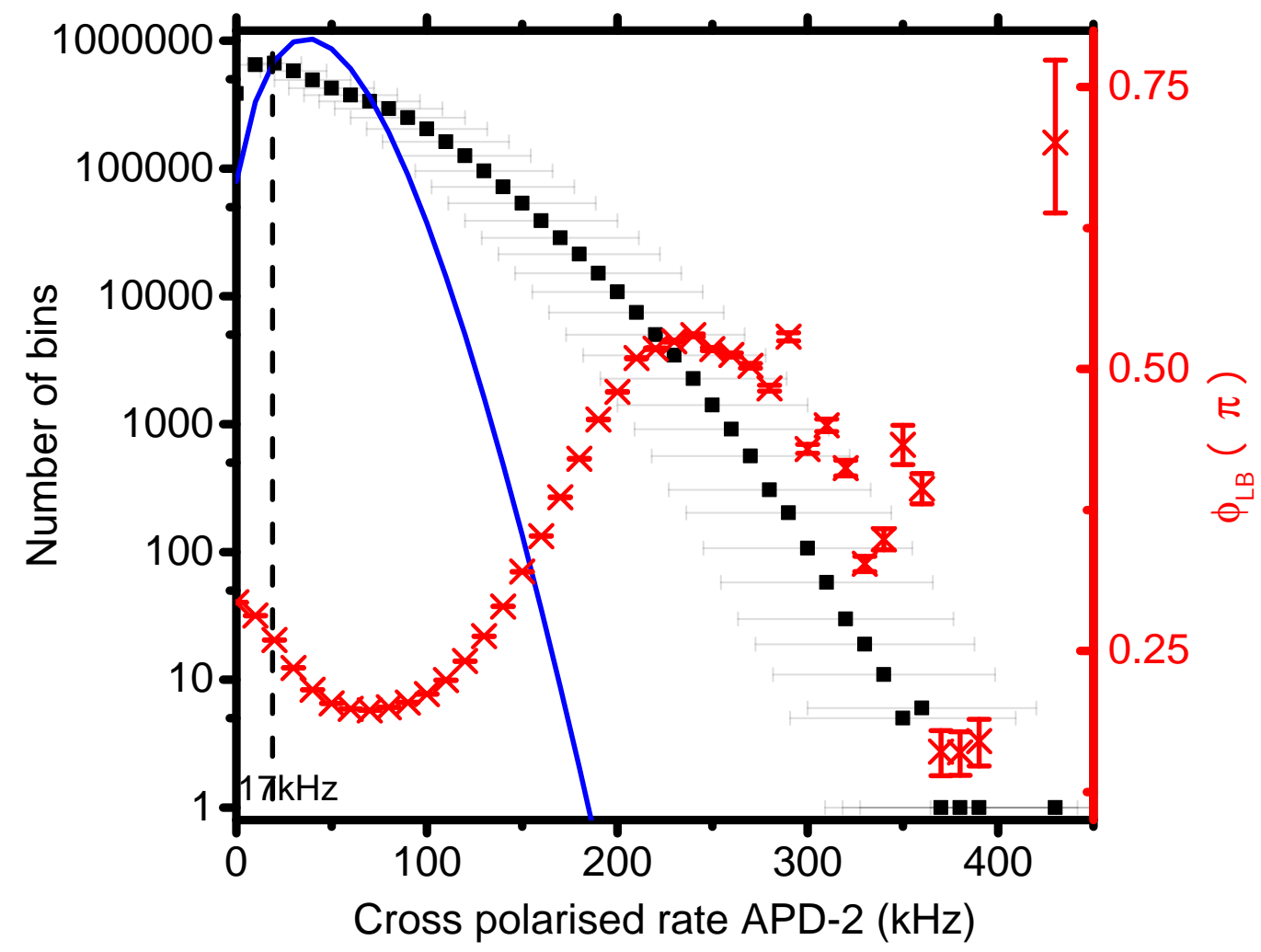

Figure 3: Frequency plot of the count-rates in APD-2 (cross-polarised) as measured in $100 \mu s$ bins (black curve) left axis, including the uncertainty in the obseved count rate (grey bars), the blue line shows the expected bin distribution based on Poissonian statistics with a $42 \mathrm{kHz}$ count rate as observed on resonance for $1 \mathrm{sec}$ integration, the dashed line shows the peak in the measured bin distribution. Red crosses displays the corresponding lower bound phase shift (right axis) measured by Eq.1 using APD-4 (H polarised) and APD-3 (V polarised)

The experiment is performed by collecting the reflected signal and splitting it into two with a nonpolarizing beamsplitter (BS), both of which are then analysed using a polarising beam splitter in the $|V\rangle$ and $|H\rangle$ basis (see Fig. 2). The two arms are statistically independent, with the first (APD-1 and APD-2) measuring the photons to "condition" those windows where the QD remains on-resonance, effectively monitoring the evolution of the environment of the QD (charge and nuclear spin configurations). Cross- 
polarized counts are only observed when the laser is close to resonance with the QD and scattered photons undergo a Faraday rotation with a non-zero projection onto $H$. In order to analyse the data the time tags of detected photons are separated into $100 \mu \mathrm{s}$ bins. Each of these time bins will have a different number of photon counts depending on the short timescale detuning between the QD and incident laser. Fig. 3 shows a histogram for the distribution of count-rates in APD-2 $(H)$ for $100 \mu s$ bin-widths (note the logarithmic scale). The vast majority of time-bins contain low count-rates, around $17 k H z$. This corresponds to the QD transition shifted away from resonance with the input laser due to jitter. Incidentally, this is in line with measurements performed far from the QD resonance, that show almost no cross-polarized counts, demonstrating no measurable ellipicity from the passive cavity.

Nevertheless, there are time-bins with significantly higher count-rates. This becomes clear by comparing the measured time bin distribution (black squares Fig.3), to the expected purely Poissonian time bin distribution (blue line Fig.3), based on the measured one second average resonant count rate in APD-2 ( $\sim 42 k H z$, corresponding to a low averaged Faraday rotation). It is clear that one should not expect to observe any time bins with a count rate above $200 \mathrm{kHz}$. The time bins that we do measure with count rates above $200 \mathrm{kHz}$ therefore represent instances where the QD and laser have remained close to resonance for sufficient time to be detected on a $100 \mu s$ interval. Thus, the more complex underlying short time dynamics are revealed using this "conditioning" technique, where each $100 \mu s$ count rate recorded at APD-2 will correspond to a specific configuration of the local environment of the QD, giving rise to a QD-laser detuning.

The second arm (APD-3, APD-4), is separated without affecting the state of the light and thus it may be used to independently evaluate the phase shift. A lower bound value for the phase shift $\left(\cos \left(\phi_{L B}\right)\right)$, that does not take into account photon ellipticity (see supporting information for full details) but that provides a lower bound for the phase, can be calculated via:

$$
\cos \left(\phi_{L B}\right)=\frac{V-H}{V+H}
$$

where in the limit that $\left|r_{R}\right| \sim\left|r_{L}\right|$ the ellipticity in the polarisation of the reflected light will be mini- 
mum, and as such $\cos \left(\phi_{L B}\right) \sim \cos (\phi)$.

Time bins with the same count rate in the conditioning arm APD-2 are grouped together. In these groups of time bins we then sum the number of counts in APD-3 as well as the counts in APD-4. This summing leads to a total number of $\mathrm{V}$ polarised counts (APD-3), and a total number of $\mathrm{H}$ polarised counts (APD-4), which are now labelled according to the count rate in the conditioning arm (APD2). Each individual time bin could be used to make an estimation of the phase shift, however large Poissonian errors on APDs-3,4 prevent this from being reliable. The summing technique we use allows us to increase the statistical significance of the value evaluated.

One expects the phase shift measured according to Eq. 2 using the counts in APD-3 $(V)$ and APD$4(H)$ to be high when correlated with specific high values of APD-2. In Fig.3 we can clearly see that as the number of cross-polarised counts increases, so does the measured phase shift, as expected. This reaches a peak value of $\phi_{L B}=0.530 \pi \pm 0.001 \pi$ at APD-2 count-rate $240 \mathrm{kHz}$. The low count rate region $(<100 \mathrm{kHz})$ is also comprised of instances where the input laser does not couple into the setup due to mechanical instabilities. Hence here we observe a non-zero phase shift that depends on the balance between the dark counts from the individual detectors used. The Poissonian noise $( \pm \sqrt{N}$, where $N$ the counts in APD-2 for a $100 \mu s$ time-window), from this then extends into the data, but becomes less significant as the number of cross polarised counts increases. At around $100 \mathrm{kHz}$ (10 counts per $100 \mu s$ ) this effect from the dark counts is negligible and no longer manifests itself in the phase measurement. In the high count rate region $(>300 \mathrm{kHz})$ we observe a drop off for the phase shift value, instead of the expected increase. This is caused by lack of statistics, for example the last point has an error of \pm 0.1 . This is expected as these last few points correspond to a vanishingly small number of bins (even just one in some cases) and any phase shift value even the error bars themselves are indeed unreliable. Further, some of the data at very high count rates is comprised of only a few or even single time bins making them outliers, which we do not use as reliable measurements. The measured phase shift is ultimately limited by the accuracy with which we can condition using APD-2. At $240 \mathrm{kHz}$ we detect 24 photons per bin, corresponding to a significant Poissonian noise on the $x$-axis 
value in Fig.3 $( \pm \sqrt{N})$.

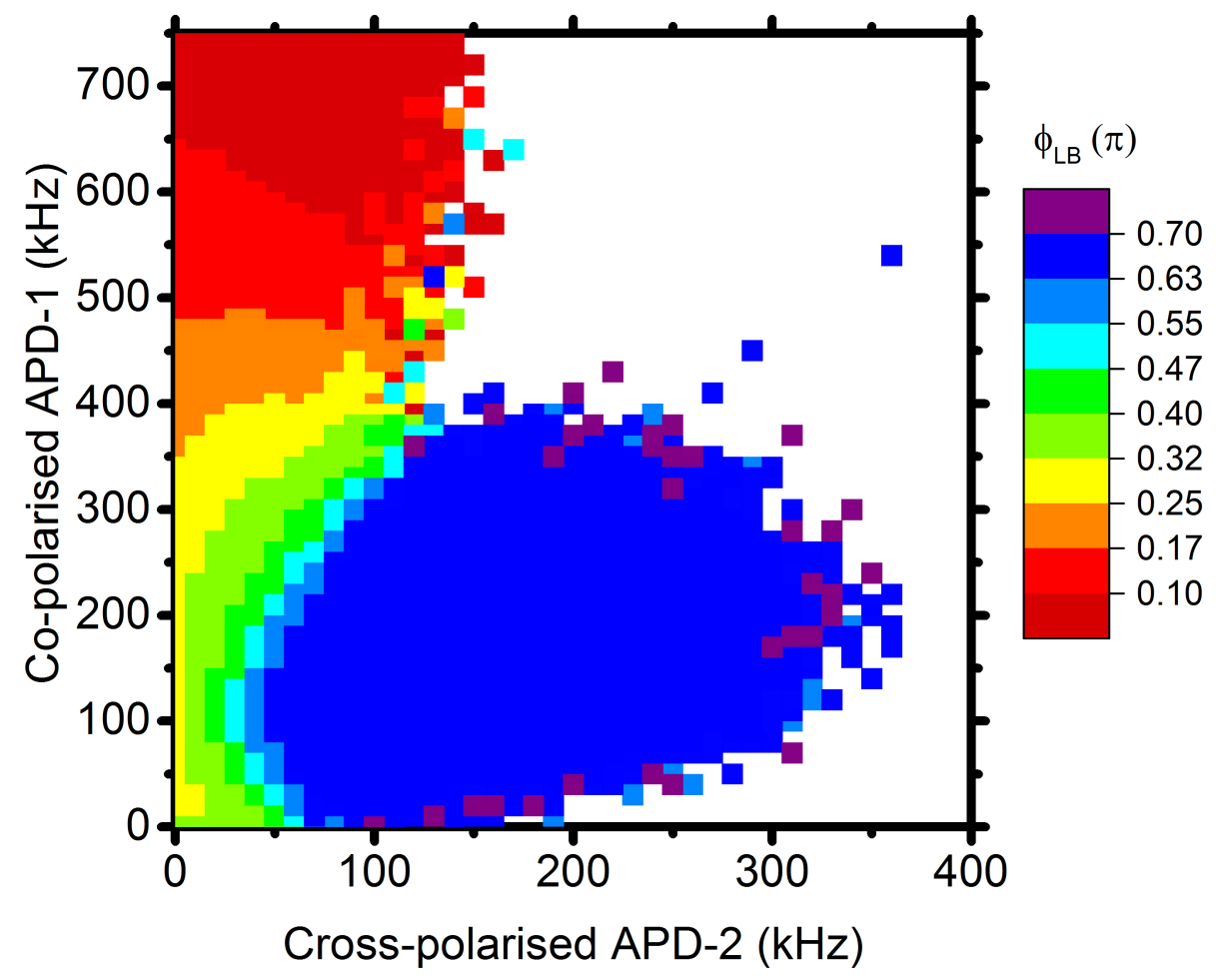

Figure 4: A plot of the number of the lower bound phase shift for specific count-rates in detectors APD-1 and APD-2 in $100 \mu$ s time-bins. The colour map now represents the QD induced phase shift measured via detectors APD-3, and APD-4.

The data in Fig. 3 is obviously limited by Poissonian noise on the x-axis due to limited statistics from APD-2. One can gain more information about when the QD and laser are resonant by implementing a two factor conditioning technique. Efficient coherent scattering from the QD will result in light rotating from $|V\rangle \rightarrow|H\rangle$, which also causes a significant intensity reduction in the directly reflected, V, channel (APD-1), i.e. the co-polarized counts should decrease. This should also occur just as the cross-polarized counts increase (APD-2). The anti-correlation between APD-1 and APD-2 gives a more accurate measure of when the QD is on-resonance, as it reduces the influence of uncorrelated background counts between the two channels (see supporting information).

To explore this Fig. 4 shows a 2D colour-plot showing the count-rates in the co- and cross-channels of the conditioning arm (APD-1,2), against the lower bound value of the phase shift $\left(\cos \left(\phi_{L B}\right)\right)$, again for $100 \mu s$ time-bins. The phase shift is calculated using the exact same technique as above however 
time bins are now grouped according to the count rate in both APD-1 and APD-2. This allows us to now build a three dimensional plot instead of two dimensional, as in Fig. 3.

A clear pattern emerges. For time-bins where the co-polarised (APD-1) counts are high, and the cross-polarised counts (APD-2) are low (approx APD-1 > $400 k H z$, APD-2 $<50 k H z$ ), we observe a small phase shift $\phi_{L B}<0.1 \pi$. This corresponds to time bins with large QD laser detunings i.e the non-resonant response. However, there is a large region where the cross-polarised counts are high and the co-polarised counts are low (approximately, APD-2 > $100 k H z$, APD-1 $<400 k H z$ ). Here the phase shift $\phi_{L B}$ is consistently above $0.63 \pi$. This scattering occurs when the QD and laser are close to resonance.

Finding the instances where near exact resonance conditions are fulfilled allows the highest phase shift observed to be measured. We calculate that to measure a $>0.95 \pi$ phase shift, the laser should be within $\pm 15 \mathrm{neV}(\sim 3.6 \mathrm{MHz})$ of the peak of the $0.8 \mu \mathrm{eV}(\sim 193 \mathrm{MHz})$ linewidth transition. This should occur $\sim 0.1 \%$ of the time (assuming inhomogeneous broadening of a $0.8 \mu \mathrm{eV}(\sim 193 \mathrm{MHz})$ QD line to $5 \mu \mathrm{eV}(\sim 1.2 G H z))$. We also require that enough photon counts may be gathered in the time-window in question to ensure effects are above the Poissonian noise, and that the $T_{1}$ spin lifetime of the transition is longer than the time-bin used. By varying the time-bin width we determine a spin $T_{1}$ of $250 \mu \mathrm{s}$ and a spectral jitter time of $1.5 \mathrm{~ms}$ (see supporting information Fig. S10). We can reliably measure the phase shift inside the blue region. One of the most statistically significant coordinates, where the measured phase has a small error (APD-1=140 $\mathrm{kHz}$ and APD-2=150 $\mathrm{kHz}$ ) of $\phi_{L B}=0.680 \pi \pm 0.001 \pi\left(122.4^{\circ} \pm 0.2^{\circ}\right)$. Note that we have a very high level of statistical confidence $(0.001 \pi)$. This is because it occurs in 1841 individual time bins, with a total of 126209 correlated photons in channels APD-3 and APD-4 available to evaluate the phase shift. There are outlying values (purple squares) with higher phase shifts. However, they have a lower statistical confidence e.g. $0.8 \pi \pm$ $\pi / 10$. Based on a $\phi_{L B}=0.680 \pi \pm 0.001 \pi$, then we observe a Faraday rotation of $\sim 0.34 \pi\left(61.2^{\circ}\right)$. 


\section{Discussion}

When comparing the highest phase shifts obtained to those predicted in Fig. 1.(b), we observe that, if the laser is on resonance with the QD, any values between $\pi / 2$ and $\pi$ are not predicted. We therefore deduce that the $\beta$-factor is $>0.5$. Consequently, every photon that enters the cavity must undergo a phase shift of $\pi$ when on resonance. Spectral detuning would reduce the phase shift, however, one would expect that some time windows represent the very close to resonance case, showing a $\pi$-phase shift. The question remains as to why we do not observe a larger phase shift consistently in any of the time-windows. For the device to be useful in a quantum information context the photons must undergo a full $\phi=\pi$, leading to a Faraday rotation of $\pi / 2$, which represents a full rotation of the polarisation from $\mathrm{V}$ to $\mathrm{H}$. There are two possible reasons for this. Either inherent losses in the system are transforming the reflected light into an elliptical state; or uncoupled background, light that is focused by the microscope objective that does not couple into the mode of the micropillar, is reducing the observed phase shift. Using white light reflectivity we measure $\eta_{\text {top }}>0.9$ (see supporting information), thus in either of these scenarios we can set a lower bound $\beta>0.6$. In fact, we believe the actual $\beta$-factor to be higher, and the main limitation is due to mode mismatch between the micropillar output mode from the top (which is Gaussian-like $(\mathrm{HE} 11)^{43}$ ) and the bulk collection optics. If we assume that the largest reliable value of $\sim 0.680 \pi$ occurs when exactly on-resonance where one would expect no co-polarized counts, the measured ratio of co/total counts $\sim 0.22$ implies that $\sim 20 \%$ of the photons arise from the background. This corresponds to a mode-matching of numerical apertures of $\sim 0.9$ (see supporting information for details). Thus, when the detuning is close to zero we infer that $80 \%$ of the reflected photons in the collection channel have interacted with the QD and undergo a deterministic $\pi$ phase shift i.e. an almost full rotation from $\mathrm{V}$ to $\mathrm{H}$. This is the first time this threshold has been directly observed in semiconductor systems, surprisingly with the use of a low Q-factor structure.

Practically, the fact that these large phase shifts can be observed indicates that spectral jitter can be overcome in our measurements, simply by collecting sufficient photons over a timescale where the QD is stable. Note that the conditioning arm here does not work in the traditional sense, where one would 
use it to pull out correlations from the data after they have been measured (post selection). In our case the first few photons in a time bin can potentially be used to predict that the rest of the photons (up to $\sim 100)$ within $100 \mu s$ will all pick up a deterministic phase shift and may be used in subsequent quantum information protocols. The significant challenge remaining is efficient mode-matching of the pillar to external optics. High fidelity gate operations will require exceptional reduction in background scatter implying that $>99 \%$ optical mode-matching is required. By integrating these micropillars, perhaps directly with optical fibre, one could potentially reflect and collect $10^{4}$ high fidelity (near full rotation) photons per $100 \mu \mathrm{s}$ when on-resonance. This would allow one to imprint a deterministic phase shift on each individual photon, entangling it with the spin to produce a $\geq 20$ photon cluster state, this is subject to the coherence time of the spin $\left(T_{2}\right)$ being sufficient long. Depending on the input rate this represents a creation rate of several times per second. Further, one would be able to actively identify when the resonance condition has been fulfilled, and switch subsequent photons into a photonic circuit using relatively slow $(100 \mathrm{kHz})$ low loss optical switches, that are currently available. This represents an immediate leap forward for the number of photonic qubits available for quantum information applications.

In summary, using a low Q-factor, high efficiency QD-microcavity system, one may achieve deterministic photon-spin interactions, by inducing a $\pi$-phase shift on a narrow bandwidth single photon. Not only do we measure, to our knowledge, by far the largest ever photon phase shift from a solid-state quantum emitter, we reach the crucial measured threshold, for the presence of deterministic interactions, of $\phi>\pi / 2 .{ }^{14}$ Further we do this in a device that has a high extraction efficiency $>90 \%$. Also, we have shown that the spectral jitter in these systems can be slow $\left(10^{5}\right.$ times slower than the exciton lifetime) and meaning that, in principle, one can trigger on time-windows where the QD line is stable. This may still be some distance away from a fully deterministic device (no photon loss and a deterministic phase shift), where $\beta$ and $\eta$ are unity, but it is still a viable regime to generate long cluster states at a reasonable rate. This represents an efficient deterministic photon-emitter interaction for output photons and experimentally demonstrates that the one-dimensional atom regime is viable and efficient 
in a scalable solid-state platform.

\section{Acknowledgement}

The authors would like to thank X.Ai and H.F. Hofmann for helpful discussions. This work was funded by the Future Emerging Technologies (FET)-Open FP7-284743 [project Spin Photon Angular Momentum Transfer for Quantum Enabled Technologies (SPANGL4Q)] and the German Ministry of Education and research (BMBF) and Engineering and Physical Sciences Research Council (EPSRC) (EP/M024156/1, EP/N003381/1 and EP/M024458/1). J.J.H. was supported by the Bristol Quantum Engineering Centre for Doctoral Training, EPSRC grant EP/L015730/1. We acknowledge the GW4 network for funding of A.Y.

\section{Supporting Information Available}

Experimental details and sample information, characterisation of the structure, theory background, supporting data.

\section{References}

(1) Ding, X.; He, Y.; Duan, Z. C.; Gregersen, N.; Chen, M. C.; Unsleber, S.; Maier, S.; Schneider, C.; Kamp, M.; Höfling, S.; Lu, C.-Y.; Pan, J.-W. On-Demand Single Photons with High Extraction Efficiency and Near-Unity Indistinguishability from a Resonantly Driven Quantum Dot in a Micropillar. Physical Review Letters 2016, 116, 020401-.

(2) Somaschi, N. et al. Near-optimal single-photon sources in the solid state. Nat Photon 2016, 10, $340-345$.

(3) Unsleber, S.; He, Y.-M.; Gerhardt, S.; Maier, S.; Lu, C.-Y.; Pan, J.-W.; Gregersen, N.; Kamp, M.; 
Schneider, C.; Höfling, S. Highly indistinguishable on-demand resonance fluorescence photons from a deterministic quantum dot micropillar device with $74 \%$ extraction efficiency. Opt. Express 2016, 24, 8539-8546.

(4) Bennett, A. J.; Lee, J. P.; Ellis, D. J. P.; Meany, T.; Murray, E.; Floether, F. F.; Griffths, J. P.; Farrer, I.; Ritchie, D. A.; Shields, A. J. Cavity-enhanced coherent light scattering from a quantum dot. Science Advances 2016, 2.

(5) Hu, C. Y.; Young, A.; O’Brien, J. L.; Munro, W. J.; Rarity, J. G. Giant optical Faraday rotation induced by a single-electron spin in a quantum dot: Applications to entangling remote spins via a single photon. Phys. Rev. B 2008, 78, 085307.

(6) Turchette, Q. A.; Hood, C. J.; Lange, W.; Mabuchi, H.; Kimble, H. J. Measurement of Conditional Phase Shifts for Quantum Logic. Phys. Rev. Lett. 1995, 75, 4710-4713.

(7) Hu, C. Y.; Munro, W. J.; Rarity, J. G. Deterministic photon entangler using a charged quantum dot inside a microcavity. Phys. Rev. B 2008, 78, 125318.

(8) $\mathrm{Hu}, \mathrm{C}$. Y.; Rarity, J. G. Loss-resistant state teleportation and entanglement swapping using a quantum-dot spin in an optical microcavity. Phys. Rev. B 2011, 83, 115303.

(9) Waks, E.; Vuckovic, J. Dipole Induced Transparency in Drop-Filter Cavity-Waveguide Systems. Phys. Rev. Lett. 2006, 96, 153601.

(10) Bonato, C.; Haupt, F.; Oemrawsingh, S. S. R.; Gudat, J.; Ding, D.; van Exter, M. P.; Bouwmeester, D. CNOT and Bell-state analysis in the weak-coupling cavity QED regime. Phys. Rev. Lett. 2010, 104, 160503.

(11) Duan, L.-M.; Kimble, H. J. Scalable Photonic Quantum Computation through Cavity-Assisted Interactions. Phys. Rev. Lett. 2004, 92, 127902. 
(12) Lindner, N. H.; Rudolph, T. Proposal for Pulsed On-Demand Sources of Photonic Cluster State Strings. Phys. Rev. Lett. 2009, 103.

(13) Auffeves-Garnier, A.; Simon, C.; Gerard, J.-M.; Poizat, J.-P. Giant optical nonlinearity induced by a single two-level system interacting with a cavity in the Purcell regime. Physical Review A (Atomic, Molecular, and Optical Physics) 2007, 75, 053823.

(14) Hofmann, H. F.; Kojima, K.; Takeuchi, S.; Sasaki, K. Optimized phase switching using a singleatom nonlinearity. Journal of Optics B: Quantum and Semiclassical Optics 2003, 5.

(15) Young, A. B.; Oulton, R.; Hu, C. Y.; Thijssen, A. C. T.; Schneider, C.; Reitzenstein, S.; Kamp, M.; Höfling, S.; Worschech, L.; Forchel, A.; Rarity, J. G. Quantum-dot-induced phase shift in a pillar microcavity. Physical Review A 2011, 84, 011803-.

(16) De Santis, L.; Antón, C.; Reznychenko, B.; Somaschi, N.; Coppola, G.; Senellart, J.; Gómez, C.; Lemaître, A.; Sagnes, I.; White, A. G.; Lanco, L.; Auffèves, A.; Senellart, P. A solid-state singlephoton filter. Nature Nanotechnology 2017, 12, 663 EP -, Article.

(17) Androvitsaneas, P.; Young, A. B.; Schneider, C.; Maier, S.; Kamp, M.; Höfling, S.; Knauer, S.; Harbord, E.; Hu, C. Y.; Rarity, J. G.; Oulton, R. Charged quantum dot micropillar system for deterministic light-matter interactions. Physical Review B 2016, 93, 241409-.

(18) Reiserer, A.; Ritter, S.; Rempe, G. Nondestructive Detection of an Optical Photon. Science 2013, $342,1349-1351$.

(19) Reiserer, A.; Kalb, N.; Rempe, G.; Ritter, S. A quantum gate between a flying optical photon and a single trapped atom. Nature 2014, 508, 237-240, Letter.

(20) Tiecke, T. G.; Thompson, J. D.; de Leon, N. P.; Liu, L. R.; Vuletic, V.; Lukin, M. D. Nanophotonic quantum phase switch with a single atom. Nature 2014, 508, 241-244, Letter. 
(21) O’Shea, D.; Junge, C.; Volz, J.; Rauschenbeutel, A. Fiber-Optical Switch Controlled by a Single Atom. Phys. Rev. Lett. 2013, 111, 193601.

(22) Bakker, M. P.; Ruytenberg, T.; Löffler, W.; Barve, A.; Coldren, L.; van Exter, M. P.; Bouwmeester, D. Quantum dot nonlinearity through cavity-enhanced feedback with a charge memory. Phys. Rev. B 2015, 91, 241305.

(23) Javadi, A.; Söllner, I.; Arcari, M.; Hansen, S. L.; Midolo, L.; Mahmoodian, S.; Kirsanske, G.; Pregnolato, T.; Lee, E. H.; Song, J. D.; Stobbe, S.; Lodahl, P. Single-photon non-linear optics with a quantum dot in a waveguide. Nature Communications 2015, 6, $8655 \mathrm{EP}$-, Article.

(24) Fushman, I.; Englund, D.; Faraon, A.; Stoltz, N.; Petroff, P.; Vuckovic, J. Controlled Phase Shifts with a Single Quantum Dot. Science 2008, 320, 769-772.

(25) Sun, S.; Kim, H.; Solomon, G. S.; Waks, E. A quantum phase switch between a single solid-state spin and a photon. Nat Nano 2016, 11, 539-544, Article.

(26) Antón, C.; Hilaire, P.; Kessler, C. A.; Demory, J.; Gómez, C.; Lemaître, A.; Sagnes, I.; LanzillottiKimura, N. D.; Krebs, O.; Somaschi, N.; Senellart, P.; Lanco, L. Tomography of the optical polarization rotation induced by a single quantum dot in a cavity. Optica 2017, 4, 1326-1332.

(27) Snijders, H.; Frey, J. A.; Norman, J.; Bakker, M. P.; Langman, E. C.; Gossard, A.; Bowers, J. E.; van Exter, M. P.; Bouwmeester, D.; Löffler, W. Purification of a single-photon nonlinearity. Nature Communications 2016, 7, 12578 EP -, Article.

(28) Daveau, R. S.; Balram, K. C.; Pregnolato, T.; Liu, J.; Lee, E. H.; Song, J. D.; Verma, V.; Mirin, R.; Nam, S. W.; Midolo, L.; Stobbe, S.; Srinivasan, K.; Lodahl, P. Efficient fiber-coupled singlephoton source based on quantum dots in a photonic-crystal waveguide. Optica 2017, 4, 178-184.

(29) Hallett, D.; Foster, A.; Hurst, D.; Royall, B.; Kok, P.; Clarke, E.; Itskevich, I.; Fox, A.; Skolnick, M.; Wilson, L. Nonlinear quantum optics with an electrically tunable quantum dot in a nano-photonic waveguide. arXiv preprint arXiv:1711.00682 2017, 
(30) Arcari, M.; Söllner, I.; Javadi, A.; Lindskov Hansen, S.; Mahmoodian, S.; Liu, J.; Thyrrestrup, H.; Lee, E. H.; Song, J. D.; Stobbe, S.; Lodahl, P. Near-Unity Coupling Efficiency of a Quantum Emitter to a Photonic Crystal Waveguide. Phys. Rev. Lett. 2014, 113, 093603.

(31) Dewhurst, S. J.; Granados, D.; Ellis, D. J. P.; Bennett, A. J.; Patel, R. B.; Farrer, I.; Anderson, D.; Jones, G. A. C.; Ritchie, D. A.; Shields, A. J. Slow-light-enhanced single quantum dot emission in a unidirectional photonic crystal waveguide. Applied Physics Letters 2010, 96, 031109.

(32) Laucht, A.; Pütz, S.; Günthner, T.; Hauke, N.; Saive, R.; Frédérick, S.; Bichler, M.; Amann, M.C.; Holleitner, A. W.; Kaniber, M.; Finley, J. J. A Waveguide-Coupled On-Chip Single-Photon Source. Phys. Rev. X 2012, 2, 011014.

(33) Reithmaier, J.; et al., Strong coupling in a single quantum dot-semiconductor microcavity system. Nature 2004, 432, 197-200.

(34) Yoshie, T.; Scherer, A.; Hendrickson, J.; Khitrova, G.; Gibbs, H. M.; Rupper, G.; Ell, C.; Shchekin, O. B.; Deppe, D. G. Vacuum Rabi splitting with a single quantum dot in a photonic crystal nanocavity. Nature 2004, 432, 200-203.

(35) Arnold, C.; Demory, J.; Loo, V.; Lemaître, A.; Sagnes, I.; Glazov, M.; Krebs, O.; Voisin, P.; Senellart, P.; Lanco, L. Macroscopic rotation of photon polarization induced by a single spin. Nat Commun 2015, 6.

(36) Young, A. B.; Hu, C. Y.; Rarity, J. G. Generating entanglement with low-\$Q\$-factor microcavities. Physical Review A 2013, 87, 012332-.

(37) Maier, S.; Gold, P.; Forchel, A.; Gregersen, N.; Mørk, J.; Höfling, S.; Schneider, C.; Kamp, M. Bright single photon source based on self-aligned quantum dot-cavity systems. Optics Express 2014, 22, 8136-8142.

(38) Gregersen, N.; Kaer, P.; Mørk, J. Modeling and Design of High-Efficiency Single-Photon Sources. IEEE Journal of Selected Topics in Quantum Electronics 2013, 19, 1-16. 
(39) Stephens, A. M.; Evans, Z. W. E.; Devitt, S. J.; Greentree, A. D.; Fowler, A. G.; Munro, W. J.; O’Brien, J. L.; Nemoto, K.; Hollenberg, L. C. L. Deterministic optical quantum computer using photonic modules. Phys. Rev. A 2008, 78, 032318.

(40) Kuhlmann, A. V.; Prechtel, J. H.; Houel, J.; Ludwig, A.; Reuter, D.; Wieck, A. D.; Warburton, R. J. Transform-limited single photons from a single quantum dot. Nat Commun 2015, 6 .

(41) Jahn, J.-P.; Munsch, M.; Béguin, L.; Kuhlmann, A. V.; Renggli, M.; Huo, Y.; Ding, F.; Trotta, R.; Reindl, M.; Schmidt, O. G.; Rastelli, A.; Treutlein, P.; Warburton, R. J. An artificial Rb atom in a semiconductor with lifetime-limited linewidth. Phys. Rev. B 2015, 92, 245439.

(42) Thyrrestrup, H. et al. Quantum Optics with Near-Lifetime-Limited Quantum-Dot Transitions in a Nanophotonic Waveguide. Nano Letters 2018, 18, 1801-1806, PMID: 29494160.

(43) Ctistis, G.; Hartsuiker, A.; van der Pol, E.; Claudon, J.; Vos, W. L.; Gérard, J.-M. Optical characterization and selective addressing of the resonant modes of a micropillar cavity with a white light beam. Phys. Rev. B 2010, 82, 195330. 